DOI $10.35381 / \mathrm{cm} . v 7 i 1.532$

\title{
Eficacia de las leyes y de los organismos de control de la contratación pública ecuatoriana
}

Efficacy of the laws and control bodies of Ecuadorian public procurement

Astrid Carolina Campoverde-Briones

dq.astridccb14@uniandes.edu.ec

Universidad Regional Autónoma de los Andes, Quevedo

Ecuador

https://orcid.org/0000-0002-3086-3347

José Fabián Molina-Mora

docentetp43@uniandes.edu.ec

Universidad Regional Autónoma de los Andes, Quevedo

Ecuador

https://orcid.org/0000-0002-2653-2721

Wilson Eduardo Castro-Núñez

uq.wilsoncastro@uniandes.edu.ec

Universidad Regional Autónoma de los Andes, Quevedo

Ecuador

https://orcid.org/0000-0001-8233-0510

Recibido: 01 de mayo de 2021

Aprobado: 01 de agosto de 2021 


\title{
RESUMEN
}

El objetivo general de la presente investigación fue analizar jurídicamente la presencia del delito de sobreprecios en la contratación pública ecuatoriana. La misma se desarrolló desde el paradigma cuantitativo, con el apoyo metodológico desde un tipo descriptivo documental con diseño bibliográfico lo cual permitió realizar un recorrido, centrada en el desarrollar un análisis jurídico. Con la utilización de la técnica de análisis de contenido, el cual aparece a lo largo de toda la investigación, permitió el estudio de la información recopilada, se empleó como instrumento de investigación la ficha o guía de análisis documental. Se concluye que el delito de sobreprecios en el Sistema Nacional de Contratación Pública se materializa debido a los vacíos legales contenidos en la Ley Orgánica del Sistema Nacional de Contratación Pública y de su organismo rector la SERCOP por no garantizar el cumplimiento de los principios constitucionales de eficacia, transparencia calidad, responsabilidad ambiental y social.

Descriptores: Corrupción; administración pública; legislación económica; tribunal administrativo; control presupuestario. (Palabras tomadas de Tesauro UNESCO).

\begin{abstract}
The general objective of this research was to legally analyze the presence of the crime of overpricing in Ecuadorian public procurement. It was developed from the quantitative paradigm, with the methodological support from a descriptive documentary type with bibliographic design, which allowed a tour, focused on developing a legal analysis. With the use of the content analysis technique, which appears throughout the entire investigation, it allowed the study of the information collected, the document analysis file or guide was used as a research instrument. It is concluded that the crime of overpricing in the National Public Procurement System materializes due to the legal gaps contained in the Organic Law of the National Public Procurement System and its governing body, SERCOP, for not guaranteeing compliance with the constitutional principles of effectiveness, transparency, quality, environmental and social responsibility.
\end{abstract}

Descriptors: Corruption; public administration; economic legislation; administrative court; budget control (words takenfrom UNESCO Thesaurus). 


\section{INTRODUCCIÓN}

La contratación pública puede definirse como la adquisición de bienes, servicios y ejecución de obras, por parte de las entidades que conforman el sector público, cuando estas presentan necesidades internas o asuntos de atención externa dentro del ámbito de sus competencias, como por ejemplo, la atención a los diferentes servicios públicos o de necesidad pública ya sea mediante la ejecución de obras, o de mantenimiento y readecuación de las infraestructuras ya existentes; todo esto a través de procedimientos establecidos por la Ley Orgánica del Sistema Nacional de Contratación Pública, donde participan personas naturales o jurídicas, del sector público como del sector privado, que tengan la calidad de proveedores mediante la habilitación del Registro Único de Proveedores (RUP) emitido por el ente rector de la contratación pública como lo es el Servicio Nacional de Contratación Pública (SERCOP), con la finalidad de alcanzar el bien común, utilizando para esto fondos públicos que son de administración del Estado, para cubrir los costos de dichas necesidades.

Por ello la contratación pública es fundamental económicamente tanto a nivel nacional como internacional. Los sistemas de contratación pública repercuten de forma significativa en el uso eficiente de los fondos públicos, sin pretermitir, el ejercicio de la eficacia de la administración pública, por ello el logro de una buena relación entre calidad y precio que, a su vez, comprende un derecho de los usuarios y consumidores, contenido en la Constitución de la República del Ecuador (2008), como se tipifica a continuación:

Artículo 52.-Las personas tienen derecho a disponer de bienes y servicios de óptima calidad y a elegirlos con libertad, así como a una información precisa y no engañosa sobre su contenido y características.

La ley establecerá los mecanismos de control de calidad y los procedimientos de defensa de las consumidoras y consumidores; y las sanciones por vulneración de estos derechos, la reparación e indemnización por deficiencias, daños o mala calidad de bienes y servicios, y por la interrupción de los servicios públicos que no fuera ocasionada por caso fortuito o fuerza mayor. 
Artículo 54.-Las personas o entidades que presten servicios públicos o que produzcan o comercialicen bienes de consumo, serán responsables civil y penalmente por la deficiente prestación del servicio, por la calidad defectuosa del producto, o cuando sus condiciones no estén de acuerdo con la publicidad efectuada o con la descripción que incorpore.

Las personas serán responsables por la mala práctica en el ejercicio de su profesión, arte u oficio, en especial aquella que ponga en riesgo la integridad o la vida de las personas.

El basamento legal, toma en consideración la expresión sobre la eficiencia y eficacia de la administración pública, la primera, que comprende, el correcto manejo de los fondos públicos (eficiencia), no es otra cosa que la facilidad que debe proveer la administración pública para el correcto ejercicio de los derechos de las personas; y, la segunda, comprende al ámbito de gestión y campo de acción de la administración pública (eficacia) que, no es otra cosa que la potestad y la atribución de la administración pública establecida en la ley, sobre lo que debe de hacer y/o ejecutar en el ejercicio de sus funciones. En este sentido, De La Torre Lascano y Quiroz Peña (2020), manifiestan que:

La falta de transparencia, control y la pérdida de institucionalidad son elementos firmes, constantes, en ese turbio esquema denominado fraude, fraguados para obtener cuantiosos beneficios personales. Ensombrecedor escenario que reseña un dimensionamiento general acerca de este fenómeno que no hace sino ratificar el enorme problema existente que se presenta a nivel mundial. (p.149)

Por ende el ejercicio de funciones para una correcta atención y servicio de la administración pública, está relacionado directamente con la contratación pública, toda vez que siempre se requerirá de la misma, para la ejecución de obras y la adquisición de bienes y servicios, como se ha señalado al inicio del presente trabajo, debiendo aquella contratación pública gozar del acceso público a la información relativa a todo el proceso de sustanciación y más aún en los contratos públicos, esto, por estricta observancia a los principios de legalidad, trato justo, igualdad, calidad, vigencia tecnológica, oportunidad, concurrencia, transparencia, publicidad; y, 
participación nacional previsto en el artículo 4 de la Ley Orgánica del Sistema Nacional de Contratación Pública(2008).

De acuerdo a Graycar y Prenzler (2013), citado por Cabezas Albán y Paredes Aulestia (2020):

Más allá de enfocarse en una definición estática, indican que la corrupción debe ser examinada en función de cuatro componentes: tipos, actividades, sectores y lugares. Los tipos se encuentran relacionados con las conductas que el derecho positivo sanciona, por ejemplo, el cohecho, el tráfico de influencias, la concusión. Es decir, el primer lente para observar cómo un Estado se encuentra tratando la corrupción necesariamente pasa por analizar cuáles son las conductas que el derecho positivo se encuentra sancionando. Con relación a las actividades, los autores sugieren que el espacio en el cual la corrupción se desarrolla debe ser evaluado para comprender cómo se está presentando e interactuando en la sociedad. Esto implica, por ejemplo, revisar si mayoritariamente ocurre en la fase de licitación de obras públicas, en la ejecución, en los pagos, en las auditorías, en el nombramiento de autoridades, en la justicia al momento de perseguir los crímenes o sentenciarlos, etc. (p.42)

En razón de lo planteado, resulta necesario indicar la importancia que tiene la contratación pública para el desarrollo de un Estado, por ello, la legislación que regula esta actividad debe ser más extensa, que tenga una capacidad de impedir el cometimiento de ilícitos a la hora de la aplicación de la contratación, pues, generalmente el índice de la corrupción por la mentada actividad, radica en el sobreprecio de la adquisición de los bienes y servicios, o en la ejecución de obras, hecho que por el mismo ordenamiento jurídico es vulnerable, toda vez que, tenemos regulación y control de precios en fármacos y productos de consumo alimenticio, no así en actividades de ejercicio profesional, como la prestación de servicios, o en la realización de obras.

A lo largo de los años en el Ecuador, han ocurrido innumerables casos de sobreprecios y demás ilícitos dentro de la contratación pública, aumentando a lo largo de la pandemia causada por el CORONAVIRUS o COVID-19 que está viviendo el mundo en la actualidad. En los primeros meses de la pandemia se desarrollaron contrataciones irregulares en el sector público que perjudicaron al Estado en más de USD 17 millones, la mayoría de los contratos se celebraron mediante invitación directa 
a proveedores a través de un régimen especial debido a la pandemia, lo cual, es permitido por el ordenamiento jurídico por tratarse de situaciones de emergencia, pero, se debe observar la condición de la emergencia para su contratación, ejemplo, por la pandemia, las contrataciones deberían ser de productos médicos, más sin embargo, se realizaron diferentes contrataciones como kits alimenticios que no tiene relación con la emergencia, se ejecutaron así mismo diferentes obras de mantenimiento, readecuación o remodelación y otras nuevas que así mismo no tuvieron relación con el objeto de la emergencia, ejemplo el implemento de carpas y establecimientos temporales para atención de pacientes afectados por el "COVID 19". (Contraloría General del Estado, 2020).

Aquellos procedimientos se practicaron arbitrariamente, inobservando las distintas fases de la contratación pública como son: preparatoria, precontractual, contractual y de ejecución, pero se escudaron en la razón de emergencia para cometer el ilícito, surgiendo a raíz de esto los sobreprecios en la mayoría de insumos médicos que diferentes entidades del Estado y del sector público adquirió y ejecutó en ese período de tiempo, generando pérdidas considerables para el Estado y por ende para las personas en general, pues se transgredió el interés general y primó el particular, el de unos cuantos. (Contraloría General del Estado, 2020).

Se planteó como objetivo de la presente investigación analizar jurídicamente la presencia del delito de sobreprecios en la contratación pública ecuatoriana.

\section{METODOLOGÍA}

La investigación se desarrolló desde el paradigma cuantitativo, con el apoyo metodológico desde un tipo descriptivo documental con diseño bibliográfico lo cual permitió realizar un recorrido, centrada en desarrollar un análisis jurídico sobre la presencia del delito de sobreprecios en la contratación pública ecuatoriana, tipificadas en las leyes y otras normas de la República. Con la utilización de la técnica de análisis de contenido, el cual aparece a lo largo de toda la investigación, permitió el estudio de la información recopilada, se empleó como instrumento de investigación la ficha o guía de análisis documental, lo que contribuyó a extraer las ideas y contrastarlas. 


\section{RESULTADOS}

El año 2020 trajo consigo uno de los peores escenarios para el sistema de salud pública a nivel mundial, la aparición de un nuevo tipo de Síndrome Respiratorio Agudo Grave, denominado COVID-19, o como popularmente se lo conoce "CORONAVIRUS", el cual hizo que los Estados de todo el mundo desarrollaran un sistema de salud, que al menos en el Ecuador se colapsó.

La emergencia sanitaria empezó a principios del mes de marzo del 2020, durante dos meses se confinó a 17 millones de ecuatorianos a resguardarse en sus hogares, a través de la modalidad del aislamiento. Entre tanto dolor y sufrimiento, miles de servidores de la salud se exponían a contagiarse por tratar de precautelar el derecho a la salud y por ende el derecho a la vida de los ciudadanos, los hospitales carecían de insumos médicos para poder brindar la asistencia necesaria a todos los pacientes, por lo que fue imprescindible realizar contrataciones por la situación que se estaba suscitando. (Alvarado Acosta y Ortega Nieto, 2020)

Según los datos del Servicio Nacional de Contratación Pública (2020), desde el 16 de marzo hasta el 31 de julio del 2020, existen 961 entidades que realizaron 7.289 contrataciones, lo que generó un monto de $\$ 209.259 .441,58$ y se emitieron 1.621 resoluciones y 6.499 informes. Lo mencionado en las líneas precedentes, permite visualizar el panorama desde un contexto nacional de carácter general, respecto a las contrataciones por las diversas entidades que forman parte del Estado, lo que pone al descubierto la importancia que la entidad rectora cumpla a cabalidad con lo establecido en las leyes pertinentes en materia de contratación pública.

En cuanto al marco legal se muestra a continuación el siguiente cuadro donde especifica las normas respectivas. 


\section{Cuadro 1.}

Normativa legal.

\begin{tabular}{|c|c|}
\hline Apoyo legal & Contenido \\
\hline \begin{tabular}{|l} 
Constitución de la República del \\
Ecuador(2008)
\end{tabular} & $\begin{array}{l}\text { Artículo 288.- Las compras públicas } \\
\text { cumplirán con criterios de eficiencia, } \\
\text { transparencia, calidad, responsabilidad } \\
\text { ambiental y social. Se priorizarán los } \\
\text { productos y servicios nacionales, en } \\
\text { particular los provenientes de la } \\
\text { economía popular y solidaria, y de las } \\
\text { micro, pequeñas y medianas unidades } \\
\text { productivas. }\end{array}$ \\
\hline $\begin{array}{l}\text { Ley Orgánica del Sistema Nacional de } \\
\text { Contratación Pública(2008) }\end{array}$ & $\begin{array}{l}\text { Artículo 4.- Establece los principios de } \\
\text { legalidad, trato justo, igualdad, calidad, } \\
\text { vigencia tecnológica, oportunidad, } \\
\text { concurrencia, transparencia, publicidad; } \\
\text { y, participación nacional. } \\
\text { Artículo 7.- Especifica que el Sistema } \\
\text { Nacional de Contratación Pública } \\
\text { (SNCP) es el conjunto de principios, } \\
\text { normas, procedimientos, mecanismos y } \\
\text { relaciones organizadas orientadas al } \\
\text { planeamiento, programación, } \\
\text { presupuestos, control, administración y } \\
\text { ejecución de las contrataciones } \\
\text { realizadas por las Entidades } \\
\text { Contratantes. } \\
\text { Artículo 8.- Determina que el Servicio } \\
\text { Nacional de Contratación Pública junto } \\
\text { con las demás instituciones y } \\
\text { organismos públicos que ejerzan } \\
\text { funciones en materia de presupuestos, } \\
\text { planificación, control y contratación } \\
\text { pública, forman parte del Sistema } \\
\text { Nacional de Contratación Pública, en el } \\
\text { ámbito de sus competencias. } \\
\text { Artículo 10.- Indica sus atribuciones, } \\
\text { siendo estas: } \\
\text { - Asegurar y exigir el cumplimiento de los } \\
\text { objetivos prioritarios del Sistema } \\
\text { Nacional de Contratación Pública. }\end{array}$ \\
\hline
\end{tabular}


-Promover y ejecutar la política de contratación pública dictada por el Directorio.

- Establecer los lineamientos generales que sirvan de base para la formulación de los planes de contrataciones de las entidades sujetas a la presente Ley Administrar el Registro Único de Proveedores RUP.

- Desarrollar y administrar el Sistema Oficial de Contratación Pública del Ecuador, compras públicas, así como establecer las políticas y condiciones de uso de la información y herramientas electrónicas del sistema.

-Administrar los procedimientos para la certificación de producción nacional en los procesos precontractuales y de autorización de importaciones de bienes y servicios por parte del Estado.

-Establecer y administrar catálogos de bienes y servicios normalizados.

-Expedir modelos obligatorios de documentos precontractuales y contractuales, aplicables a las diferentes modalidades y procedimientos de contratación pública, para lo cual podrá contar con la asesoría de la Procuraduría General del Estado y de la Contraloría General del Estado.

- Dictar normas administrativas, manuales e instructivos relacionados con esta Ley;

- Recopilar y difundir los planes, procesos y resultados de los procedimientos de contratación pública.

- Incorporar y modernizar herramientas conexas al sistema electrónico de contratación pública y subastas electrónicas, así como impulsar la interconexión de plataformas tecnológicas de instituciones y servicios relacionados. 


\begin{tabular}{|c|c|}
\hline & $\begin{array}{l}\text {-Capacitar y asesorar en materia de } \\
\text { implementación de instrumentos y } \\
\text { herramientas, así como en los } \\
\text { procedimientos relacionados con } \\
\text { contratación pública. } \\
\text {-Elaborar parámetros que permitan } \\
\text { medir los resultados e impactos del } \\
\text { Sistema Nacional de Contratación } \\
\text { Pública y en particular los procesos } \\
\text { previstos en esta Ley. } \\
\text {-Facilitar los mecanismos a través de los } \\
\text { cuales se podrá realizar veeduría } \\
\text { ciudadana a los procesos de } \\
\text { contratación pública; y, monitorear su } \\
\text { efectivo cumplimiento. } \\
\text {-Elaborar y publicar las estadísticas del } \\
\text { Sistema Nacional de Contratación } \\
\text { Pública. } \\
\text {-Capacitar y certificar, de acuerdo a lo } \\
\text { dispuesto en el Reglamento, a los } \\
\text { servidores y empleados nombrados por } \\
\text { las entidades contratantes, como } \\
\text { operadores del Sistema Nacional de } \\
\text { Contratación Pública. } \\
\text {-Asesorar a las entidades contratantes y } \\
\text { capacitar a los proveedores del Sistema } \\
\text { Nacional de Contratación Pública sobre } \\
\text { la inteligencia o aplicación de las normas } \\
\text { que regulan los procedimientos de } \\
\text { contratación de tal sistema. } \\
\text {-Las demás establecidas en la presente } \\
\text { ley, su reglamento y demás normas } \\
\text { aplicables. }\end{array}$ \\
\hline $\begin{array}{l}\text { Ley Orgánica Reformatoria del Código } \\
\text { Orgánico Integral Penal en Materia } \\
\text { Anticorrupción(2021) }\end{array}$ & $\begin{array}{l}\text { Artículo 294.1.-Sobreprecios en } \\
\text { contratación pública. Las o los } \\
\text { servidores públicos, las personas que } \\
\text { actúen en virtud de una potestad estatal } \\
\text { en alguna de las instituciones del } \\
\text { Estado; o, los proveedores del Estado } \\
\text { que realicen arbitrariamente los } \\
\text { procesos de contratación pública con } \\
\text { evidente y comprobado sobreprecio al } \\
\text { precio ordinario establecido por el } \\
\text { mercado y determinado como tal por la }\end{array}$ \\
\hline
\end{tabular}




\begin{tabular}{|l|l|}
\hline & $\begin{array}{l}\text { Contraloría General del Estado, serán } \\
\text { sancionados con pena privativa de } \\
\text { libertad de cinco a siete años. } \\
\text { El informe de la Contraloría General del } \\
\text { Estado que determina la existencia del } \\
\text { sobreprecio en contratación pública, } \\
\text { deberá ser otorgado por dicha entidad, } \\
\text { en el plazo máximo de quince días } \\
\text { contados a partir de la fecha de la } \\
\text { solicitud efectuada por la o el fiscal. } \\
\text { Si la conducta prevista en el primer } \\
\text { párrafo ha sido cometida } \\
\text { aprovechándose de una declaratoria de } \\
\text { emergencia o estado de excepción, } \\
\text { serán sancionadas con el máximo de la } \\
\text { pena prevista. }\end{array}$ \\
\hline
\end{tabular}

Elaboración: Los autores.

\section{DISCUSIÓN}

La Constitución de la República del Ecuador, así como la Ley Orgánica del Sistema Nacional de Contratación Pública establecen que los procesos de contratación públicos deben cumplir con criterios de eficiencia, transparencia, calidad, responsabilidad ambiental y social así como deben ser regidos por principios tales como los principios de legalidad, trato justo, igualdad, calidad, vigencia tecnológica, oportunidad, concurrencia, transparencia, publicidad; y, participación nacional, disposiciones que son contrarrestadas al momento de la materialización de estos ilícitos, quedando tanto la LOSNCP como la SERCOP, impotentes a la hora de contrarrestar su cometimiento.

Por otro lado, en vista de lo que establece la Contraloría General del Estado, en lo referente a los sobreprecios en la contratación pública, es claro que ni la Ley Orgánica del Sistema Nacional de Contratación Pública ni su organismo rector, son capaces de contrarrestar el cometimiento de actos de corrupción, un claro ejemplo de que estos organismos, no cumplen con este cometido, es la inclusión en el COIP del delito de sobreprecios y demás delitos de anticorrupción, así como la inserción de un Subsistema Nacional de control conformado por Contraloría General del Estado, 
Procuraduría General del Estado, Fiscalía General del Estado, Sistema Nacional de Contratación Pública, Consejo Participación Ciudadana y Control Social y Superintendencias con el fin de efectuar un control interconectado y eficaz de cualquier situación o irregularidad que se presente en la contratación pública, denotando falencias en el anterior sistema encabezado por la SERCOP y la Ley Orgánica de Sistema Nacional de Contratación Pública, pero esta interconexión conlleva a otro problema, que es la transgresión al principio de coordinación de la administración pública, pues las competencias de estas instituciones del Estado descrita anteriormente ya no son de forma racional y ordenada, incluso existen duplicidades de funciones, en otras palabras, no existe entonces una finalidad institucional para combatir la corrupción.

\section{CONCLUSIÓN}

Se concluye que el delito de sobreprecios en el Sistema Nacional de Contratación Pública se materializa debido a los vacíos legales contenidos en la Ley Orgánica del Sistema Nacional de Contratación Pública y de su organismo rector la SERCOP, por no garantizar el cumplimiento de los principios constitucionales de eficacia, transparencia calidad, responsabilidad ambiental y social, mediante mecanismos de coordinación con otras entidades de la administración pública, dentro de sus fases que son: preparatoria, pre contractual, contractual y ejecución de la contratación pública.

\section{FINANCIAMIENTO}

No monetario.

\section{AGRADECIMIENTO}

A la Universidad Regional Autónoma de los Andes, Quevedo, por motivar el desarrollo de la Investigación. 


\section{REFERENCIAS}

Alvarado Acosta, G. y Ortega Nieto, M. (2020) Ineficacia del servicio nacional de contratación pública (SERCOP) en los contratos de insumos médicos durante la emergencia sanitaria en Ecuador. Trabajo de titulación de abogado(as). Universidad de Guayaquil. Recuperado de: https://n9.cl/q2798

Asamblea Nacional Constituyente de la República del Ecuador, (2008). Constitución de la República del Ecuador. Montecristi. Registro Oficial 449 de 20-oct-2008. Recuperado de https://n9.cl/sia

Asamblea Nacional (2021) Ley Orgánica Reformatoria del Código Orgánico Integral Penal en Materia Anticorrupción. Registro Oficial - Segundo Suplemento № 392.Recuperado de: https://n9.cl/tufqu

Cabezas Albán, V., y Paredes Aulestia, V. (2020). La corrupción en 3D: una radiografía de sus componentes. Revista de políticas y problemas públicos, 1(10), 37-51. https://doi.org/10.37228/estado comunes.v1.n10.2020.148

Congreso Nacional. (2002). Ley Orgánica de la Contraloría General del Estado. Registro Oficial 595. Quito, Ecuador

Contraloria General del Estado(2020). Contrataciones irregulares perjudican al Estado en más de USD 17 millones Recuperado de https://www.contraloria.gob.ec/CentralMedios/SalaPrensa/23870

De La Torre Lascano, C. y Quiroz Peña, J. (2020) Fraude organizacional. Percepciones previas a la creación de un observatorio del fraude Revista de temas de coyuntura y perspectivas, 5(3), 147-183. https://doi.org/10.5281/zenodo.4061902

Gobierno de la República del Ecuador (2008). Ley Orgánica del Sistema Nacional de Contratación Pública. (Registro Oficial Suplemento 395 del 04 de agosto de 2008). Recuperado de: www.lexis.com.ec

Gobierno de la República del Ecuador (2008). Servicio Nacional de Contratación pública.. Recuperado de: https://n9.cl/mcmin

Gobierno de la República del Ecuador (2020) Registro Único de Proveedores. Recuperado de: $\underline{\text { https://n9.cl/pb0adc }}$ 


\section{CIENCIAMATRIA}

Revista Interdisciplinaria de Humanidades, Educación, Ciencia y Tecnología Año VII. Vol. VII. N¹. Edición Especial. 2021 Hecho el depósito de ley: pp201602FA4721

ISSN-L: 2542-3029; ISSN: 2610-802X

Universidad Nacional Experimental Francisco de Miranda (UNEFM). Santa Ana de Coro. Venezuela

Astrid Carolina Campoverde-Briones; José Fabián Molina-Mora; Wilson Eduardo Castro-Núñez

C2021 por los autores. Este artículo es de acceso abierto y distribuido según los términos y condiciones de la licencia Creative Commons Atribución-NoComercial-Compartirlgual 4.0 Internacional (CC BY-NC-SA 4.0) (https://creativecommons.org/licenses/by-nc-sa/4.0/). 